\title{
The use of a solution of the inverse heat conduction problem to monitor thermal stresses
}

\author{
Jan Taler ${ }^{1,{ }^{*}}$ Piotr Dzierwa ${ }^{1}$, Magdalena Jaremkiewicz ${ }^{1}$, Dawid Taler $^{2}$, Karol Kaczmarski ${ }^{1}$, \\ and Marcin Trojan ${ }^{1}$ \\ ${ }^{1}$ Institute of Thermal Power Engineering, Cracow University of Technology, Cracow, Poland \\ ${ }^{2}$ Institute of Thermal Engineering and Air Protection, Faculty of Environmental Engineering, Cracow, \\ Poland
}

\begin{abstract}
Thick-wall components of the thermal power unit limit maximum heating and cooling rates during start-up or shut-down of the unit. A method of monitoring the thermal stresses in thick-walled components of thermal power plants is presented. The time variations of the local heat transfer coefficient on the inner surface of the pressure component are determined based on the measurement of the wall temperature at one or six points respectively for one- and three-dimensional unsteady temperature fields in the component. The temperature sensors are located close to the internal surface of the component. A technique for measuring the fastchanging fluid temperature was developed. Thermal stresses in pressure components with complicated shapes can be computed using FEM (Finite Element Method) based on experimentally estimated fluid temperature and heat transfer coefficient
\end{abstract}

\section{Introduction}

The lifetime of pressure elements and damage-free operation of the boiler can be reduced by high-rate heating or cooling of the boiler during its start-up or shutdown. During the start-up and shutdown of the boiler as well as when the boiler load changes, the permissible rate of temperature variations of the fluid should not be exceeded in so-called critical pressure components. If the rate of temperature change is less than the limit value, so the sum of stresses due to the pressure and thermal loads on the edge of the hole on the inner surface of the pressure element does not exceed the allowable stress [1]. Start-ups and shutdowns of the boiler with heating and cooling rates smaller or equal to the allowable values determined by the European standard [1] ensure safe and long-term operation of the boiler. Formulas for permissible rates of heating and cooling of thick-walled components given in the standard can be used for long-term heating or cooling of critical thick-walled components at a constant rate. In real boiler operation conditions, the rate of fluid temperature change is not constant and changes over time. More accurate methods for determining the optimum temperature variations of the fluid during heating of thick-walled boiler components, without adopting a simplifying assumption that the temperature field in the component is quasi-steady presented Taler et al. [2-3] and Dzierwa et al. [4-5]. Jaremkiewicz et al. [6-8] developed a new

\footnotetext{
*Corresponding author: taler@mech.pk.edu.pl
} 
technique for measuring transient fluid temperature by which the permissible temperature change rates or allowable temperature variations can be controlled with much higher accuracy compared to the massive industrial thermometers currently used in power plants. The fluid temperature measured with high accuracy is also necessary for determining transient thermal stresses in cylindrical pressure elements [9] or other elements with more complicated shapes. Boiler manufacturers determine thermal stresses on the inner surface of the pressure element based on the temperature measurement in the middle of the wall thickness and near the inner surface of the element. This method of measurement is inaccurate especially at rapid temperature changes of the fluid, and even in the quasi-steady state because the stresses are determined at a distance of 6 to $10 \mathrm{~mm}$ from the inner surface instead of on the inner surface. More complex methods are used to determine the thermal stresses on the inner surface of the pressure element based on the measurement of the temperature inside the wall for varying heating rates over time [10-12]. A simple method of controlling thermal stresses in cylindrical elements without a hole based on temperature measurement at two points was developed in [10]. Another method of determining thermal stresses in cylindrical elements is based on temperature measurement on the external insulated surface of the pressure element [11]. A review of the literature shows that determining and possibly increasing the permissible rates of heating and cooling of thickwalled boiler components is important both for the flexibility of the boilers and their lifetime. The analysis of previous works also indicates the lack of a method of monitoring stresses in thick-walled boiler elements at rapid changes in the temperature of the fluid. This paper first discusses the method of determining the permissible rate of temperature changes in the walls of thick-walled components assuming the quasi-steady state temperature field in the pressure element. A stable and accurate procedure for monitoring the rate of temperature changes of the component walls has also been developed. Next, the methods of monitoring thermal stresses in pressure elements used by boiler manufacturers were discussed. The paper also presents a new method for monitoring thermal stresses in pressure components of large steam boilers. The time variations of the local heat transfer coefficient on the inner surface of the pressure component are determined based on the measurement of the wall temperature at one or at six points located close to the internal surface of the component depending on whether the transient temperature field is one-dimensional or three-dimensional. The temperature and the heat flux on the inner surface of the pressure component result from the solution of the transient one or three-dimensional IHCP (Inverse Heat Conduction Problem). Transient temperature of the fluid is determined with high accuracy using the new measurement technique based on the thermometer with a new design and IHCP solution. Thermal stresses in a pressure component with a complicated shape are computed using FEM-software and experimentally estimated fluid temperature and heat transfer coefficient. Stress concentration coefficients due to pressure and thermal load are calculated using the FEM to determine the stresses at the hole edges. Numerous computational tests have been carried out to demonstrate the accuracy of the methods developed for the so-called "exact" measurement data and measurement data disturbed by random measurement errors. Also, the results of thermal stress monitoring in thick-walled cylindrical components using the actual measurement data are presented. In the stress monitoring system implemented in power plants, the rate of the wall temperature variation and the thermal stresses on the inner surface of the pressure component are determined online and compared with the permissible values. 


\section{Determining the permissible rates of temperature variations of the fluid using a quasi-steady state approach}

Using the European standard EN 12952-3 [1], allowable rate of the fluid and wall temperature changes was determined on the assumption of thermal quasi-steady state in thick wall components of the boiler to simplify the analysis. Quasi-steady state occurs after a long time of heating or cooling the element at a constant rate (Fig. 1a). The rate of wall temperature changes at quasi-steady state is the same at every point of the component wall. If the temperature distribution in the wall is one-dimensional, then thermal stresses in quasi-steady state depend only on the rate of change in fluid temperature and do not depend on time. Quasi-steady state arises when a Fourier number Fo is equal or greater than 0.5, i.e.

$$
\mathrm{Fo}^{*} \geq \sim 0.5
$$

Solving inequality (1) gives:

$$
t^{*} \geq \frac{s^{2} \mathrm{Fo}^{*}}{\kappa_{w}}
$$

where: $t^{*}$ - time after which the quasi-steady state is formed, $F o^{*}$ Fourier number after which the quasi-steady state is formed, $s$-wall thickness, $\kappa_{w}$-thermal diffusivity.

a)

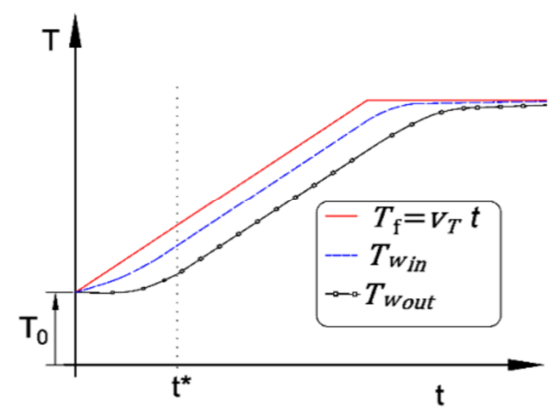

b)

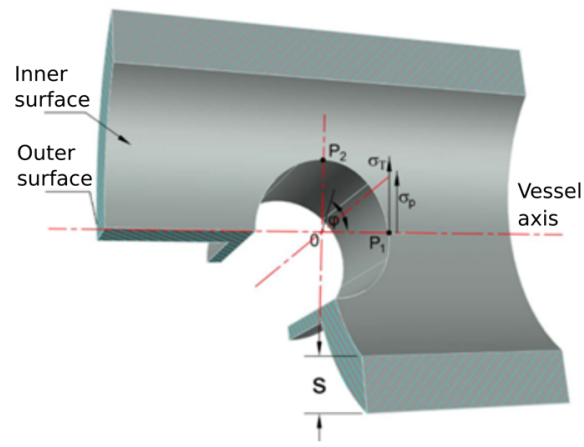

Fig. 1. Increasing the temperature of the fluid at a constant rate; (a) temperature of the fluid, internal and external surface of the cylindrical element as a function of time during the heating of the element at a constant rate to the desired design temperature, (b) cylindrical pressure element weakened by the opening.

The heating or cooling of thick-walled pressure component is to be carried out so that the circumferential stress $\sigma_{\varphi, P_{1}}$ at the hole edge at point $\mathrm{P}_{1}$, does not exceed the allowable stress $\sigma_{a l}$.

$$
\sigma_{\varphi, P_{1}} \leq \sigma_{a l}
$$

where the total circumferential stress $\sigma_{\varphi, P_{1}}$ is the sum of the stress due to pressure $\alpha_{p} \sigma_{p}$ and the stress $\alpha_{T} \sigma_{T}$ due to the temperature difference across the vessel wall

$$
\sigma_{\varphi, P_{1}}=\alpha_{p} \sigma_{p}+\alpha_{T} \sigma_{T}
$$


where: $\alpha_{p}$ - stress concentration factor for circumferential stress due to pressure ; $\alpha_{T}$ - stress concentration factor for circumferential thermal stress.

The circumferential stress $\sigma_{p}$ at the inner surface of the cylindrical vessel, caused by gauge pressure $p$ and circumferential stress $\sigma_{T}$ due to temperature difference on the wall thickness, are given by

$$
\begin{aligned}
& \sigma_{p}=\frac{p d_{m}}{2 s} \\
& \sigma_{T}=\phi_{c y l} \frac{v_{T} s^{2}}{\kappa_{w}} \cdot \frac{E_{w} \beta_{w}}{1-v_{w}}
\end{aligned}
$$

where: $p$ - pressure, $d_{m}$ - mean diameter, $v_{T}$ - the rate of temperature variation, $E_{w^{-}}$-Young's modulus, $\beta_{w}$ - linear thermal expansion coefficient, $v_{w}$ - Poisson's ratio.

So-called the shape factors $\phi_{c y l}$ for the cylindrical vessel is as follows

$$
\phi_{c y l}=\frac{\left(u_{o}^{2}-1\right)\left(3 u_{o}^{2}-1\right)-4 u_{o}^{4} \ln u_{o}}{8\left(u_{o}^{2}-1\right)\left(u_{o}-1\right)^{2}}
$$

where: $u_{o}$ - the ratio of the outer to the inner radius; $r_{\text {out }}$ - the outer radius of the component, $r_{i n}$ - the inner radius of the component.

The thermal circumferential stress $\sigma_{T}$ at the inner surface, given by Eq.(6) is equal to the axial stress provided that the cylindrical component is very long and its ends are free. The stress concentration factor $\alpha_{p}$ for circumferential stress due to pressure at the hole edge is

$$
\alpha_{p}=\frac{\sigma_{\varphi, p}}{\sigma_{p}}
$$

where: $\sigma_{\varphi, p^{-}}$circumferential stress due to pressure at the hole edge on the inner surface of the component, $\sigma_{p}$ - circumferential stress due to pressure at the inner surface of the cylindrical component without a hole. Similarly, a stress concentration factor $\alpha_{T}$ for thermal stress is defined

$$
\alpha_{T}=\frac{\sigma_{\varphi, T}}{\sigma_{T}}
$$

where: $\sigma_{\varphi, T^{-}}$circumferential thermal stress at a hole edge on the inner surface of the component, $\sigma_{T^{-}}$circumferential thermal stress at the inner surface of the cylindrical component without hole. Figures $2 \mathrm{a}$ and $\mathrm{b}$ show the unsteady distribution of temperature and stress in the outlet header of the last stage of the steam superheater. The temperature and stress concentration computation were carried out using the ANSYS v.18 software. The outlet header was made of X10CrWMoVNb9-2 (P-92 steel). The following constant material properties were adopted for the calculations : thermal diffusivity $\kappa_{w}=5.73 \times 10^{-6} \mathrm{~m}^{2} / \mathrm{s}$, Young's modulus $E_{w}=1.7 \times 10^{5} \mathrm{MPa}$, linear thermal expansion coefficient $\beta_{w}=1.88 \times 10^{-5} 1 / \mathrm{K}$, Poisson's ratio $v_{w}=0.3$. The heating rate was $v_{T}=4 \mathrm{~K} / \mathrm{min}$. The heat transfer coefficient on the inner surface of the element was $h=1000 \mathrm{~W} / \mathrm{m}^{2} \mathrm{~K}$. The outer radius is rout $=0.2095 \mathrm{~m}$, thickness $\mathrm{s}=0.1 \mathrm{~m}$. From the analysis of the results presented in Figure $2 \mathrm{a}$, it can be seen that the highest temperature has the inlet connectors the live steam due to the small thickness of the wall. You can see the thermal stress concentration on the edges of the holes (Fig. 2b), where the stresses around one hole do not affect the stresses around the adjacent hole. Coefficients of the concentration of stress on the edges of holes in the analyzed chamber are presented in Table 1. It can be seen that the concentration factor $\alpha_{p}$ is much larger at the point $\mathrm{P}_{1}$ compared to point $\mathrm{P}_{2}$. The stresses concentration factor of thermal stress $\alpha_{T}$ is in turn slightly larger at point $P_{2}$ compared to point $P_{1}$. Figure 3 shows variations in the thermal 
stress concentration factor for the analyzed header as a function of the heat transfer coefficient on its internal surface.

a)

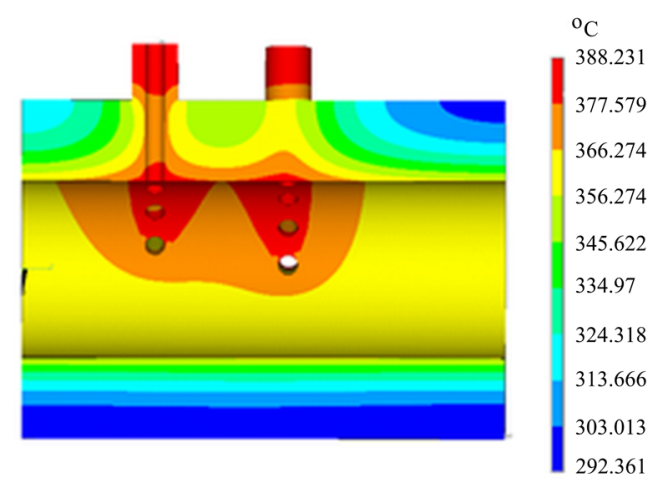

b)

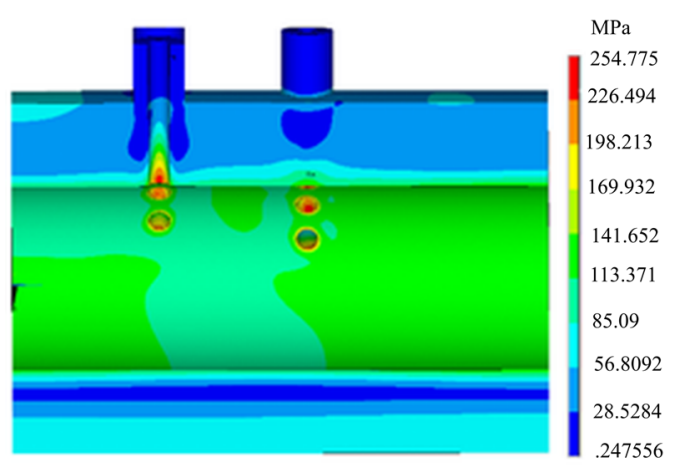

Fig. 2. Distribution of temperature (in ${ }^{\circ} \mathrm{C}$ ) and Huber-von Mises stress (in MPa) fields in outlet header of final superheater at quasi-steady state for heating rate $v_{T}=4 \mathrm{~K} / \mathrm{min}$.

From the structural analysis with Finite Element Method (FEM) it follows that during the heating of the thick-walled element, larger total circumferential stress due to pressure and temperature difference may occur at the point $\mathrm{P}_{2}$ lying on the edge of the hole (Fig. 1b). Considering that the circumferential thermal stress on the edge of the hole is compressive and the stress due to pressure is tensile, the point $\mathrm{P}_{2}$ on the edge of the hole is more be loaded during heating than point $P_{1}$. This results from the lack of adequate compensation at point $P_{2}$ for thermal stress by the stress caused by pressure. This means that the critical point during the heating of the thick-walled component is point $\mathrm{P}_{2}$. The admissible heating rates should, therefore, be determined from the condition of not exceeding the permissible stress at point $\mathrm{P}_{2}$. In the European standard EN12952-3 [1], this fact is not taken into account because the permissible heating and cooling rates are determined due to the allowable stress at point $\mathrm{P}_{1}$. The permissible rate of heating or cooling of the pressure element is determined from Eq.(3), in which the allowable stress is determined from the Wöhler fatigue diagram in the European standard EN12952-3 [1]. Instantaneous rates of heating or cooling of thick-walled elements can be determined on-line based on the wall temperature measurements and compared with the permissible values. If the allowable temperature variations of the wall determined with Eq.(3) are not exceeded, this means that also thermal stress on the inner surface of the element, given by Eq.(6) does not exceed the allowable value. 


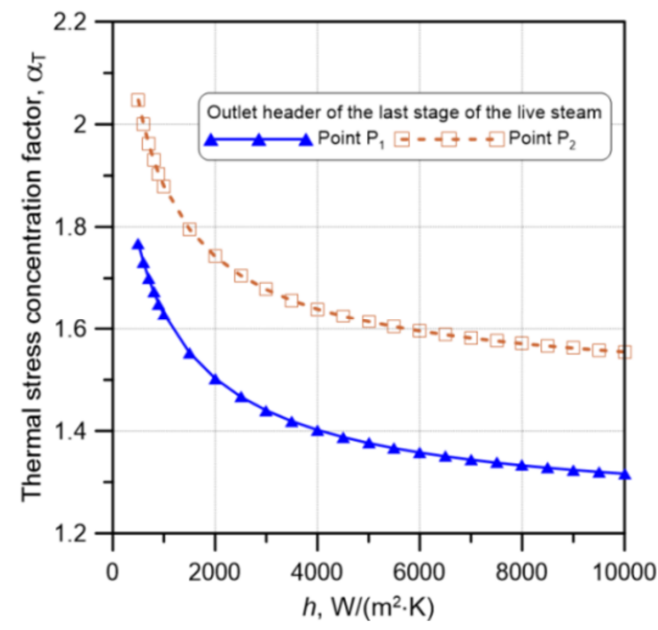

Fig. 3. Stress concentration factor $\alpha_{T}$ for thermal circumferential stress computed by FEM as a function of the heat transfer coefficient $h$ on the inner surface of the live steam outlet header.

This indirect method of stress control is approximate due to the assumption of quasisteady state in the pressure component. Another popular method of controlling thermal stresses in pressure elements used by boiler manufacturers is to measure the temperature difference between the average temperature on the wall thickness and the temperature at a point located several millimeters from the inner surface of the component.

Table 1. Stress concentration factor for circumferential stress by pressure and thermal load at quasisteady state with the heat transfer coefficient on the inner surface equal $h=1000 \mathrm{~W} / \mathrm{m}^{2} \mathrm{~K}$

\begin{tabular}{|c|c|c|c|c|}
\hline \multirow{2}{*}{} & \multicolumn{2}{|c|}{$\begin{array}{c}\text { The stress concentration factor } \\
\alpha_{p} \text { for circumferential stress } \\
\text { due to pressure }\end{array}$} & \multicolumn{2}{|c|}{$\begin{array}{c}\text { The stress concentration } \\
\text { factor } \alpha_{T} \text { for circumferential } \\
\text { thermal stress }\end{array}$} \\
\cline { 2 - 5 } & $\begin{array}{c}\text { Point } \mathrm{P}_{1} \\
\text { (FEM) }\end{array}$ & $\begin{array}{c}\text { Point } \mathrm{P}_{2} \\
\text { (FEM) }\end{array}$ & $\begin{array}{c}\text { Point } \mathrm{P}_{1} \\
\text { (FEM) }\end{array}$ & $\begin{array}{c}\text { Point } \mathrm{P}_{2} \\
\text { (FEM) }\end{array}$ \\
\hline $\begin{array}{c}\text { Outlet header of the } \\
\text { final superheater }\end{array}$ & 3.859 & 1.126 & 1.628 & 1.874 \\
\hline
\end{tabular}

\section{Monitoring of temperature variation rates and thermal stresses used by the manufacturers}

During operation of the boiler or other pressure equipment, the rate of temperature change of the critical pressure elements can be monitored so that the limit values determined from Eq. (3) are not exceeded. The rate of temperature change is the first derivative after a time from the temperature of the measured wall. Measured temperature contains random temperature measurement errors that make it very difficult to determine the heating and cooling rate correctly. Without prior filtration of the measured temperatures, the determined rates of temperature variation would contain very large oscillations. In work [13] for local smoothing of measured temperature a third degree polynomial with respect to time was used. The odd number of consecutively measured temperatures equal to $2 \mathrm{~N}+1$ was fitted with the local third-degree polynomial. 


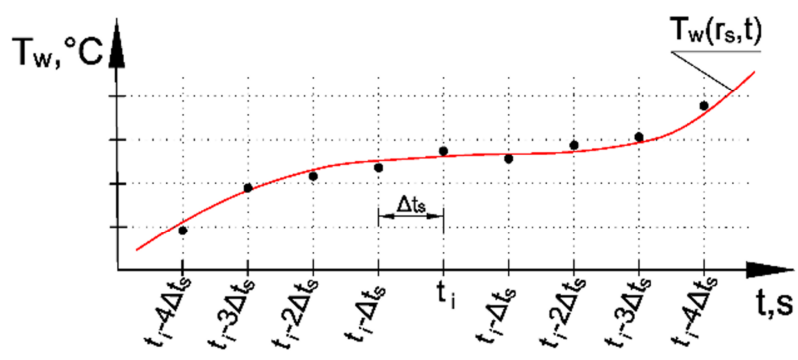

Fig. 4. Smoothing of measured temperature variations by the 9-point digital filter based on the polynomial of the third degree.

The temperature value and its derivative were determined only in the middle $t_{i}$ of the analyzed time interval of $2 N \Delta T$ width (Fig.4.). The filter is movable. In each subsequent time step, the last measurement point is rejected, and the next point added. For a 9-point filter $(N=4)$ the smoothed temperature rate is given by

$$
\begin{aligned}
& \mathrm{v}_{T}\left(r_{s}, t_{i}\right)=\left.\frac{d T_{w}\left(r_{s}, t\right)}{d t}\right|_{t=t_{i}}=\frac{1}{1188 \Delta t_{s}}\left[86 T_{w}^{m}\left(r_{s}, t_{i}-4 \Delta t_{s}\right)-142 T_{w}^{m}\left(r_{s}, t_{i}-3 \Delta t_{s}\right)-\right. \\
& 193 T_{w}^{m}\left(r_{s}, t_{i}-2 \Delta t_{s}\right)-126 T_{w}^{m}\left(r_{s}, t_{i}-\Delta t_{s}\right)+126 T_{w}^{m}\left(r_{s}, t_{i}+\Delta t_{s}\right)+193 T_{w}^{m}\left(r_{s}, t_{i}+\right. \\
& \left.\left.2 \Delta t_{s}\right)+142 T_{w}^{m}\left(r_{s}, t_{i}+3 \Delta t_{s}\right)-86 T_{w}^{m}\left(r_{s}, t_{i}+4 \Delta t_{s}\right)\right], \quad t_{i} \geq 4 \Delta t_{s}(10)
\end{aligned}
$$

where : $r_{s^{-}}$the location of the thermocouple, $t$ - time, $\Delta t_{s^{-}}$the sampling time of the wall temperature measurement. It is also possible to use a 7-point filter $(\mathrm{N}=3)$ when the random errors are small, or an 11-point filter $(\mathrm{N}=5)$ [14] when measurement errors are higher. Boiler manufacturers use Eq.(4) to calculate circumferential stresses at the edge of the opening. The pressure stress $\sigma_{p}$ occurring in Eq.(4) is first calculated using Eq.(5) assuming that the cylindrical element is not weakened by the holes. In practice, it is enough to measure only the pressure as a function of time to determine the circumferential stress $\sigma_{p}$ due to pressure at the inner surface of the component. The stress $\sigma_{p}$ is defined by Eq.(5) for the cylindrical wall. The thermal circumferential stress $\sigma_{T}$ at the inner surface of the component without holes is given by the exact formula

$$
\sigma_{T}=\frac{E_{w} \beta_{w}}{1-v_{w}}\left[T_{w, m}(t)-T_{w}\left(r_{i n}, t\right)\right]
$$

where $T_{w}(r, t)$ is an integral mean wall temperature on the wall thickness, defined as

$$
T_{w, m}(t)=\frac{2}{r_{\text {out }}^{2}-r_{\text {in }}^{2}} \int_{r_{\text {in }}}^{r_{\text {out }}} T_{w}(r, t) r d r
$$

where the symbol $T_{w}(r, t)$ denotes the wall temperature.

Because of the wall temperature distribution $T_{w}(r, t)$ is not known from the measurement, so the boiler manufacturers assume that the average wall temperature $T_{w, m}(t)$ is equal to the wall temperature $T_{w}(r, t)$ measured in the middle of its thickness, and the temperature of the inner surface $T_{w}\left(r_{i n}, t\right)$ in Eq.(11) is replaced by the temperature $T_{w}\left(r_{i n}+\delta, t\right)$ of the wall measured in the distance $\delta$ from its inner surface (Fig.5). The distance $\delta$ of the temperature measurement point from the internal surface is usually from 6-10 $\mathrm{mm}$. Due to the high pressure inside the element, the smaller distance $\delta$ is usually unacceptable. 


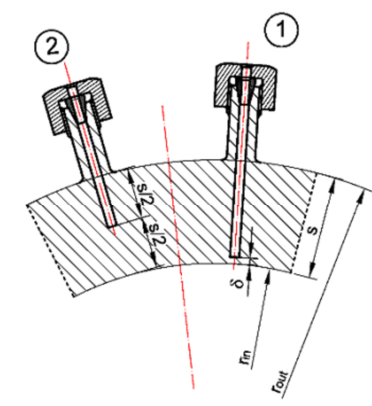

Fig. 5. Location of thermocouples for measurement of cylindrical wall temperature; 1- temperature sensor located near the inner surface of the component, 2-temperature sensor located at the centre of the wall.

However, the accuracy of the simplified method of monitoring circumferential thermal stresses on the inner surface of the component used by boiler manufacturers is insufficient. In work [15] it was shown that when measuring the element temperature at a distance $\delta=6 \mathrm{~mm}$ from the inner surface of the pressure element with a thickness of $s=100 \mathrm{~mm}$, the relative error of determining circumferential thermal stress at the inner surface was $\varepsilon=17.16 \%$ even in the quasi-steady state. In the case of rapid changes in fluid temperature, this error will be even greater.

\section{Thermal stress monitoring based on the solution of the inverse heat conduction problem}

With rapid changes in the temperature of the fluid, the temperature sensor should be located near the inner surface of the thick-walled element at a short distance of several millimeters. By placing the thermocouple near the inner surface, quick changes in the fluid temperature are not too strongly attenuated at the measurement location, so that the actual internal surface temperature and the heat flux can be reproduced. The inverse heat conduction problem (IHCP) in a thick-wall cylindrical element was solved with the following assumptions:

- the unsteady temperature field is one-dimensional

- thermo-physical properties of the component material, such as specific heat, density, and the thermal conductivity are depending on the temperature

- the thermocouple for measuring the wall temperature is located in the distance of the $\delta$ from the inner surface of the element, and the heat flux or temperature is known (Fig. 6) on the outer surface of the component

- The fluid's transient temperature is measured with high accuracy using a new design thermometer. 


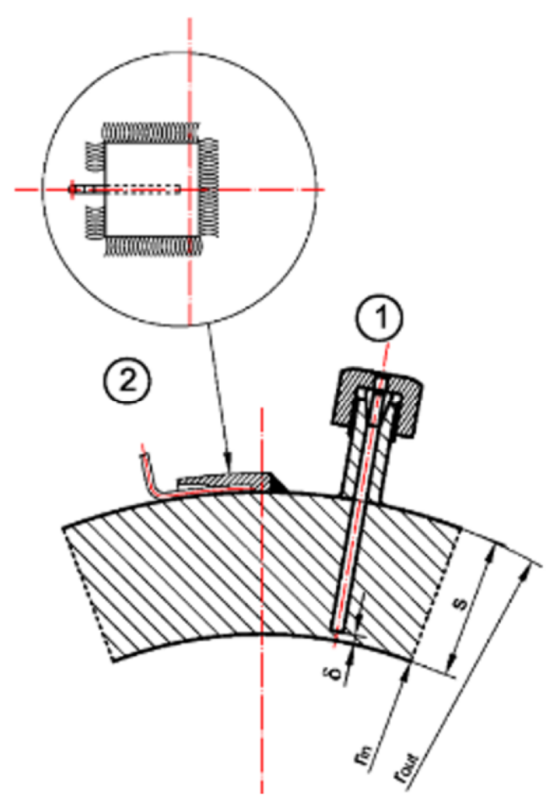

Fig. 6. The indirect method of determining the thermal stress on the inner surface of a cylindrical element by measuring the wall temperature at a distance $\delta$ from the inner surface (location 1 ) and the outer surface temperature (location 2).

The temperature and the heat flux on the inner surface of the component are determined from the IHCP solution. The transient temperature of the fluid is known from the measurement so that it is possible to determine the coefficient of heat transfer on the internal surface. Since the housings of industrial thermometers are massive [16], the method presented in [6-8] has been applied to determine the fluid temperature to reduce the dynamic errors of temperature measurements. Knowing the heat transfer coefficient and the temperature of the fluid, the thermal stresses in the thick-walled pressure component can be determined using own programs or using commercial programs based on the FEM (Finite Element Method) method. For components of complex shapes, the heat transfer coefficient can be determined on that part of the component where the temperature field is onedimensional, e.g., for cylindrical components with openings, the heat transfer coefficient will be determined at such a distance that the openings do not disturb the one-dimensional temperature field.

\section{Determining the temperature, heat flux, and heat transfer coefficient at the inner surface of the cylindrical component from the solution of the inverse heat transfer problem}

Transient heat conduction equation for the cylindrical wall is as follows

$$
\rho_{w} c_{w} \frac{\partial T_{w}}{\partial t}=\frac{1}{r} \frac{\partial}{\partial r}\left[r k_{w}\left(T_{w}\right) \frac{\partial T_{w}}{\partial r}\right]
$$

The partial differential equation (1) is subject to the following initial and boundary conditions 


$$
\begin{aligned}
& \left.T_{w}\right|_{t=0}=T_{w 0} \\
& \left.T_{w}\right|_{r=r_{i n}+\delta}=f(t)
\end{aligned}
$$

If the outer surface of the element is perfectly thermally insulated, the boundary condition on this surface has the following form

$$
\left.k_{w} \frac{\partial T_{w}}{\partial r}\right|_{r=r_{\text {out }}}=0
$$

Due to the easy accessibility to the external surface, the temperature of the surface is sometimes measured. In this case, the boundary condition for the external surface is given by

$$
\left.T_{w}\right|_{r=r_{\text {out }}}=g(t)
$$

The transient temperature distribution in the direct area $\left(r_{i n}+\delta\right) \leq r \leq r_{\text {out }}$ was determined using the finite volume method (FVM) [17, 18]. The direct area was divided into $N-2$ finite volumes. A system of $(N-1)$ or $(N-2)$ ordinary differential equations for node temperatures was obtained which was solved using the Runge - Kutta method of the fourth order. From the solution of the direct problem the temperature distribution $T_{w}(r, t)$ in the region $\left(r_{i n}+\delta\right)$ $\leq r \leq r_{\text {out }}$ was obtained, i.e., the wall temperature at the nodes $2-(N+1)$ was known (Fig. 7).

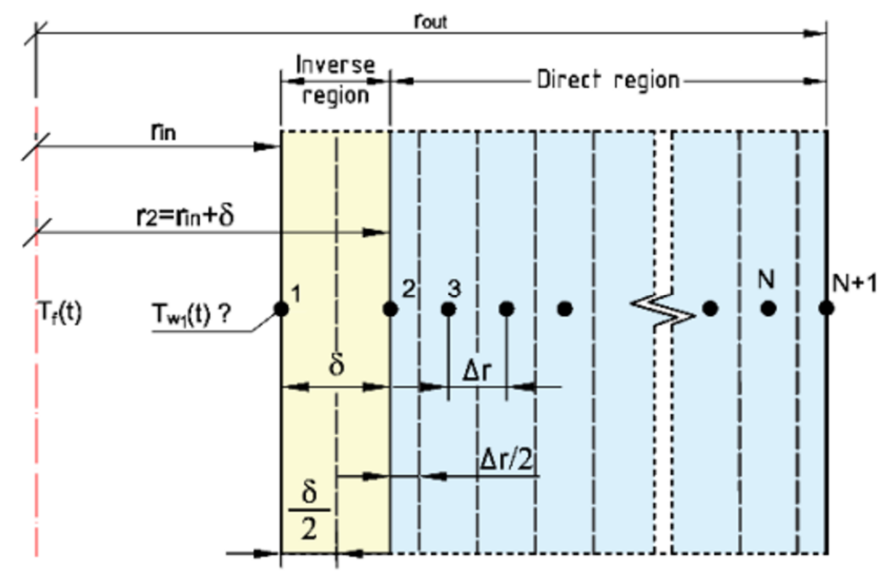

Fig. 7. Division of the cylindrical wall into finite volumes for determining temperature distribution in the direct region $\left(r_{\text {in }}+\delta\right) \leq r \leq r_{\text {out }}$, and inverse region $r_{\text {in }} \leq r \leq\left(r_{\text {in }}+\delta\right), \Delta r=\left[r_{\text {out }}-\left(r_{\text {in }}+\delta\right)\right] /(N-1)$

Next, an IHCP was solved. The temperature at the node 2 (Fig.7) was known from measurement and temperature at node 3 was determined by solving the direct heat conduction problem in the region $\left(r_{\text {in }}+\delta\right) \leq r \leq r_{\text {out }}$.

\section{Conclusions}

The method of determining the permissible rate of temperature changes of the thickwalled components assuming the quasi-steady state temperature field in the pressure components was presented. Stress concentration coefficients due to pressure and thermal load were calculated using the finite element method to determine the stresses at the hole edges.

A stable and accurate procedure for monitoring the rate of temperature changes of the component walls has also been developed. Also, the methods of monitoring thermal stresses 
in pressure elements used by boiler manufacturers were discussed. Due to their low accuracy, they can only be used to estimate the thermal stresses roughly.

\section{References}

1. European Standard EN 12952-3, Water-tube boilers, and auxiliary installations. Part 3: Design and calculation for pressure parts, European Committee for Standardization. Brussels (2001)

2. J. Taler, P. Dzierwa, Optimum heating of pressure vessels with holes. ASME J. Pressure Vessel Technology; 137, 011202-1-8 (2014)

3. J. Taler, P. Dzierwa, A new method for optimum heating of steam boiler pressure components, Int. J. Energ. Res., 35, 897-908 (2011)

4. P. Dzierwa, Optimum heating of pressure components of steam boilers with regard to thermal stresses, J. Therm. Stresses, 39, 874-886 (2016)

5. P. Dzierwa, M. Trojan, D. Taler, K. Kamińska, and J. Taler, Optimum heating of thickwalled pressure components assuming a quasi-steady state of temperature distribution, J. Therm. Sci., 25, 380-388 (2016)

6. M. Jaremkiewicz, D. Taler, T. Sobota, Measurement of transient fluid temperature, Int. J. Therm. Sci., 87, 241-250 (2015)

7. M. Jaremkiewicz, Accurate measurement of unsteady state fluid temperature, Heat Mass Transfer, 53, 887-897 (2017)

8. M. Jaremkiewicz, J. Taler, Measurement of transient fluid temperature in a pipeline, Heat Transfer Eng., 39, issues 13-14, 1227-1234 (2018)

9. D. Taler, K. Kaczmarski, Mathematical modelling of the transient response of pipeline, J. Therm. Sci., 25, 549-557 (2016)

10. J. Taler, W. Zima, M. Jaremkiewicz, Simple method for monitoring transient thermal stresses in pipeline, J. Therm. Stresses, 39, 386-397 (2016)

11. J. Taler, B. Węglowski, and M. Pilarczyk, Monitoring of thermal stresses in pressure components using inverse heat conduction methods, Int. J. Numer. Method. H., 27, 740756 (2017)

12. J. Taler, P. Dzierwa, D. Taler, M. Jaremkiewicz, M. Trojan, Monitoring of thermal stresses and heating optimization including industrial applications, Nova Science Publishers, New York (2016)

13. J. Taler, Exact solution of inverse heat conduction problems, Encyclopedia of Thermal Stresses, 3, Springer, 1440-1465 (2014)

14. J. Taler, D. Taler, Measurement of heat flux and heat transfer coefficient, Heat flux: processes, measurement techniques, and applications, 1-103, Nova Science Publishers, New York, (2012)

15. J. Taler, D. Taler, K. Kaczmarski, P. Dzierwa, M.Trojan, T. Sobota, Monitoring of thermal stresses in pressure components based on the wall temperature measurement, Energy, 160 500-519 (2018)

16. ASME PTC 19.3 TW-2010, Thermowells. Performance Test Codes, An American National Standard, The American Society of Mechanical Engineers, New York (2010)

17. A. Cebula, D. Taler, Finite volume method in heat conduction, Encyclopedia of thermal stresses, 4 F-G, Springer, 1645-1658 (2014)

18. J. Taler, A semi-numerical method for solving inverse heat conduction problems, Heat Mass Transfer, 31, 105-111 (1996) 